Taula redona: La vehiculació de la romanística a través de les revistes

Glessgen, M D ; Roques, G ; Holtus, G ; Veny, J ; García Mouton, P ; Varvaro, A ; Trotter, D

DOI: https://doi.org/10.1515/9783110299892.101

Posted at the Zurich Open Repository and Archive, University of Zurich

ZORA URL: https://doi.org/10.5167/uzh-79536

Book Section

Published Version

Originally published at:

Glessgen, M D; Roques, G; Holtus, G; Veny, J; García Mouton, P; Varvaro, A; Trotter, D (2013). Taula redona: La vehiculació de la romanística a través de les revistes. In: Casanova Herrero, E; Calvo Rigual, C. Actas del XXVI Congreso Internacional de Lingüística y de Filología Románicas, 6-11 septiembre 2010, Valencia. Berlin: De Gruyter, 101-122.

DOI: https://doi.org/10.1515/9783110299892.101 


\section{Taula redona: \\ La vehiculació de la romanística a través de les revistes ${ }^{1}$}

\section{Mise en perspective (par Martin-D. Glessgen) ${ }^{2}$}

\subsection{Le problème de l'évaluation dans la Science}

Notre débat a pour objet les revues de la romanistique et, plus précisément, les problèmes de l'évaluation qui sont intrinsèques à la production, à la diffusion et à la réception des revues. Le choix de cette thématique a été motivé par la montée invraisemblable d'évaluations de toute sorte à laquelle nous avons assistés dans l'univers académique ces dernières années. Actuellement sont évalués, partout dans le monde, les cursus d'études, les instituts, les groupes de recherches, les universités entières, les chercheurs individuels, leurs publications et, également, les revues. Ce sont des phénomènes très récents que nous n'avions pas encore rencontrés dans les années 1990 et qui auraient été tout simplement inimaginables dans les années 1980, au moins dans les Sciences humaines et en Europe. En tant que directeur de la Revue de Linguistique Romane, je suis confronté à cette problématique depuis 2006, quand les premières vagues d'évaluation des revues ont apparu.

La question est très complexe et elle contient, au moins, deux aspects contradictoires:

(i) D'un côté, l'évaluation est, bien entendu, un principe pleinement constitutif de la Science. Elle est indispensable pour ses finalités et pour sa mise en œuvre quotidienne. Tout chercheur et tout étudiant qui choisit de traiter un thème doit choisir d'emblée les méthodes à suivre et les publications sur lesquelles il fondera son propre travail: ce sont là des actes d'évaluation qui reposent sur des jugements de valeur et de qualité. Aucun choix thématique ni méthodologique ne peut se passer d'une interrogation évaluative; aucune extraction de données n'est possible sans l'intervention d'un jugement et, donc, d'une évaluation. L'évaluation est par conséquent inévitable, indispensable et positive pour la Science.

(ii) De l'autre côté, la tendance fortement politisée des évaluations à outrance que nous connaissons présente de grands dangers pour le bon fonctionnement de l'univers académique. Prenons l'exemple des revues romanistes: nous sommes tous d'accord que le ladin, le valencien, le roumain, le français et l'espagnol du Mexique ont la même valeur épistémique; de la même manière, la morphologie, la phonétique, la syntaxe ou la lexicologie sont toutes indispensables pour la linguistique, même si aucun d'entre

1 La sessió ha estat coordinada per Martin-Dietrich Glessgen (Universitat de Zuric) i per Emili Casanova (Universitat de València). El contingut d'aquesta taula redona ha estat publicat amb anterioritat (amb lleugeres diferències) a la Revue de Linguistique Romane (RLiR 295-296 [2010], 565-588), amb el títol Les revues de la romanistique et les problèmes de l'évaluation.

2 La Table ronde a été conçue par Emili Casanova et Martin-D. Glessgen lors de la préparation du Congrès; la mise en œuvre concrète est restée aux soins de ce dernier, étant donné la charge de travail considérable de l'organisateur principal du Congrès pendant l'été 2011. 
nous ne domine pleinement la tradition d'études dans tous les domaines et pour tous les idiomes romans. Il est pourtant évident qu'une revue dédiée essentiellement à la description et l'interprétation grammaticales du français standard contemporain sera considérée par les évaluations de type quantitatif comme plus importante qu'une revue qui traite les divers domaines linguistiques de l'occitan ou l'histoire du lexique ou encore la toponymie romane. Du coup, les bibliothèques universitaires qui orienteront de plus en plus leurs achats d'après les catégories $\langle A-B-C\rangle$ cesseront d'acheter des revues qui sont fondamentales pour les langues dites mineures et pour les sujets autres que la grammaire. Pire encore, les jeunes chercheurs ne voudront plus publier que dans les revues de type 〈A〉 pour ne pas nuire à leur carrière: ce sont donc des cercles vicieux. En dernière instance, la diversité qui fait toute la force de notre Science pourrait se voir amputée et les évaluations qui ont été créées pour améliorer le fonctionnement de l'univers académique risquent d'avoir précisément l'effet contraire. Des techniques qui peuvent fonctionner dans les sciences exactes peuvent ne pas fonctionner du tout dans les sciences de l'Homme, et il serait possible que ce soit ici le cas.

Il nous semble important de soumettre à la réflexion les fondements et les implications des différentes formes d'évaluation liées aux revues. Cela permet alors de mieux distinguer les aspects positifs et indispensables de l'évaluation scientifique de ses aspects politisés et irrationnels.

\subsection{L'évaluation dans les revues}

Le rôle des revues dans le fonctionnement de la Science est bien connu. Les revues ont une meilleure diffusion que les monographies et, surtout, elles garantissent un élément de qualité par l'évaluation à laquelle les articles publiés sont soumis. Par ailleurs, les revues contiennent des comptes rendus qui sont d'une grande utilité pour pouvoir s'orienter dans un univers scientifique multiforme. Il faut, en effet, distinguer clairement le domaine des articles de celui des comptes rendus.

Les articles ont toujours connu une attention particulière de type évaluatif. Toute décision sur l'acceptation, le refus ou la réélaboration d'un texte est le résultat d'une réflexion évaluative, souvent intense. Dans le passé, cette réflexion a été le rôle du seul directeur de la revue; la qualité d'une revue comme la Zeitschrift für romanische Philologie à travers le $\mathrm{XX}^{\mathrm{e}}$ siècle aurait été impensable sans la personnalité scientifique d'exception qu'était Walther von Wartburg. Aujourd'hui, des comités de lecture et des évaluateurs externes interviennent pour garantir la qualité des articles publiés (Peer Review). Ces évaluations sont souvent anonymes dans les deux sens (les évaluateurs ne connaissent pas l'identité de l'auteur et viceversa). Dans le cas de notre Revue, ce principe n'est pas facile à appliquer: aucun scientifique compétent n'aurait pu avoir le moindre doute sur l'auteur, dans le dernier fascicule, lors de la lecture de l'article «Standardisation, koinéisation et l'historiographie du français» (RLiR, 295-296, 5, 26) ou encore des «Brèves remarques sur le Trésor dóu Felibrige de Frédéric Mistral» (RLiR, 295-296, 199-214). Il est évident que nous n'indiquons pas aux auteurs des articles le nom des évaluateurs, mais, même là, la discussion scientifique entre les spécialistes pourrait s'avérer productive.

Le cas est différent pour les comptes rendus. Contrairement aux articles, leur vocation première est de type évaluatif; mais, toujours contrairement aux articles, les comptes rendus 
eux-mêmes ne font pas l'objet d'évaluations systématiques; par ailleurs, et c'est la troisième différence, si les articles sont normalement proposés par leurs auteurs à une revue donnée, les comptes rendus sont demandés, pour la plupart, par la revue même.

Bien entendu, les directeurs d'une revue et le comité de rédaction peuvent proposer certaines adaptations aux auteurs de comptes rendus et ils doivent empêcher toute forme d'abus dans ce genre délicat; mais les interventions sont beaucoup plus limitées que dans le cas des articles.

En ligne générale, les revues remplissent bien leur rôle d'outil d'évaluation et d'orientation dans la Science actuelle. La qualité d'une revue repose, entre autres, sur une bonne gestion des diverses tâches d'évaluation intrinsèques à ce genre. L'architecture de l'univers scientifique doit simplement accepter certains déséquilibres structurels: si les monographies connaissent une évaluation après leur publication -par les comptes rendus-, les articles connaissent une évaluation avant leur publication -par les responsables des revues-. Enfin, les comptes rendus eux-mêmes et le travail des responsables des revues ne sont pas ultérieurement évalués, au moins jusqu'il y a peu.

C'est en effet ici qu'intervient l'outil récent de l'évaluation des revues dans leur intégralité et l'on pourrait espérer que cela puisse mener à une augmentation de qualité des revues, comme c'est le cas dans les sciences exactes. Or, l'expérience semble démontrer que nos disciplines se prêtent très mal à cet exercice. Déjà, le travail pour une revue comporte beaucoup de charges et peu d'avantages pour les responsables: une évaluation positive n'a donc pas le même effet que dans les sciences exactes, où elle peut amener, par exemple, des subventions à la revue. Mais les véritables problèmes sont bien plus profonds. Les évaluations des revues portent sur des paramètres qui sont avant tout de type extérieur comme leurs thématiques, la longueur des articles et comptes rendus, la ou les langues de publication, la présence ou non de résumés etc. Par ailleurs, l'extrême diversité des sujets d'études qui sont réunis sous le large toit de la linguistique romane rend illusoire une concurrence à l'extrême: il est possible de comparer la qualité de trente études neuropsychologiques qui ont comme objet un type d'aphasie particulier; mais comment comparer un travail dialectométrique avec une étude sur les rôles sémantiques d'actants dans des dialectes italiens? comment y établir une hiérarchie?

Avec notre ami Hans Goebl nous avons développé devant ce dilemme l'idée que la métaphore adéquate pour le travail de qualité dans nos disciplines, n'est pas la pyramide, avec une base extrêmemement large et un sommet étroit, mais un type de montagne que l'on appelle en français, par un emprunt à l'espagnol, mesa ou meseta (comme les plateaux centraux de l'Espagne et du Mexique); autrement dit: une large plateforme qui accueille des productions scientifiques de qualité fortement diversifiées mais difficilement hiérarchisables entre elles.

Face à une organisation académique essentiellement compartimentée d'après les langues et littératures particulières, les revues romanistes représentent aujourd'hui l'un des facteurs décisifs de cohésion et d'innovation dans cette discipline transversale et internationale. Si l'évaluation est un phénomène omniprésent et indispensable dans ces revues, je suis convaincu que la tendance politique actuelle des évaluations calquées sur les sciences exactes est extrêmement dangereuse pour notre Science. Cette conviction est encore accentuée par les réflexions suivantes qui émanent de scientifiques qui ont tous une grande expérience en tant que directeurs d'une revue romaniste et qui ont accepté de développer ici les aspects de la question qui leur semblent les plus importants. 


\section{La Revue de Linguistique Romane (par Gilles Roques)}

Je voudrais apporter ici un témoignage, celui de l'ancien directeur de la Revue de Linguistique Romane. Comme mes prédécesseurs, je ne me suis jamais occupé ni d'un Comité de lecture, ni de l'évaluation de la Revue. A la différence de la plupart des autres revues scientifiques, nous ne dépendons que de nous-mêmes, c'est-à-dire de vous, les membres de notre Société. Nous avons acquis cette indépendance par votre soutien constant, par notre impeccable gestion financière et administrative, fondée sur le bénévolat. Notre tradition constante a toujours été d'accepter les articles de nos membres, «sans distinction d'aucune sorte que ce soit, à condition que les études proposées soient de haut niveau scientifique et non de vulgarisation», selon la formule répétée dans le rapport moral de tous les SecrétairesAdministrateurs successifs. Le directeur de la Revue, qui pouvait consulter un spécialiste, était en dernier ressort le seul juge du haut niveau scientifique requis. C'est qu'en fait la Revue était ouverte à des Professeurs reconnus, dont certains voire même beaucoup, à tort ou à raison, auraient jugé saugrenue l'idée qu'on pût examiner la valeur scientifique de leur article. La sélection se faisait plutôt en aval. Straka et moi-même nous efforcions de publier un maximum d'articles de haut niveau, afin que les sociétaires ne proposent à la Revue que le meilleur de leur production. Ceci, joint à la réputation de sévérité qui nous était faite, explique qu'un écrémage à la source s'opérait naturellement.

Les choses ont changé quand ont publié dans la Revue des chercheurs en quête de reconnaissance. Et la Revue n'a pas manqué ce virage souhaitable, car gage de renouvellement. Il serait trop facile de citer les noms de jeunes chercheurs, devenus des maîtres éminents et dont les tous premiers travaux ont été publiés dans la Revue. Passez en revue les tables des matières des articles et des comptes rendus parus dans la décennie 1980-1990! Pour ce faire, nous avons dû évoluer nous aussi sur ce point, bien qu'à contrecœur, je l'avoue. Une première brèche a été créée quand ce qui était, depuis l'origine, le Bureau de notre Société est devenu en même temps, pour sauver les apparences, le Comité scientifique de la Revue, au Congrès de Palerme, en 1995 ( $R L i R, 59,648$ et p. 2 de la couverture du même fascicule). Ce Comité en luimême était tellement banal (et le terme même de comité avait quelque chose du gaullien comité Théodule) que nous avions pensé que nous ne devions pas y impliquer les plus hautes autorités de la Société que sont le Président et les Vice-présidents. Mais, alors qu'A. Varvaro, alors Président, s'était fort bien accommodé de cette situation, son successeur, M. Wilmet, a tenu à les inclure dans le Comité (RLiR, 62, p. 2 de la couverture du fascicule de juillet-décembre 1998). Ce Comité scientifique s'est lui-même ensuite scindé en deux: un Comité de rédaction et un Comité scientifique ( $R L i R, 69$, p. 2 de la couverture du fascicule de janvier-juin 2005).

Naturellement, «l'évaluation est indispensable et positive pour la Science» comme vient de le rappeler Martin Glessgen. Heureusement que nous jugeons constamment ce que nous faisons, sans quoi nous ne serions pas des scientifiques. Nous jugeons aussi les travaux de nos étudiants, de nos collègues et même de nos maîtres; Straka, qui avait connu le pire et le meilleur de l'humanité, était très attaché à la «hiérarchie des valeurs», qui allait à l'encontre de certaines idées alors très en vogue. D'autre part, les articles d'une revue ne sont pas une thèse; ils sont à la fois moins qu' une thèse, par leur dimension et par la façon dont le problème est traité, et plus par l'exigence de rigueur scientifique et de renouvellement méthodologique que le lecteur en attend. De surcroît, et peut-être principalement, une revue comme la nôtre 
est un lieu de vie, qui ne doit pas être accaparé par une école, une langue, un champ d'étude, mais doit établir des passerelles entre tous les courants et constituants des études romanes. De là la nécessité de faire place dans nos colonnes à des études variées, stimulantes pour le lecteur. Dans ce genre très particulier, le pire est le ronronnement monotone; nous sommes un peu dans la situation d'un journal, qui doit coller à l'actualité scientifique, ce qui est incompatible avec la lourdeur des procédures bureaucratiques. Dans ces conditions, il faut aussi accepter le risque de l'erreur. Il a pu m'arriver de publier un article insuffisant, je l'ai regretté, et l'on n'a pas manqué de me le dire. Mais je suis sûr d'avoir publié d'excellents articles qui auraient été refusés par un comité de lecture.

Lieu de vie et hiérarchie des valeurs trouvent leur complète illustration dans les comptes rendus que publie une revue. Il ne s'agit pas de publier cinq ans après sa sortie, le compte rendu détaillé d'un ouvrage, sur lequel la plupart de ses utilisateurs les plus concernés se sont fait eux-mêmes leur opinion, mais d'exprimer à chaud une réaction spontanée et motivée. Dans notre revue, c'est une conquête qui s'est faite progressivement. A l'origine, il n'y avait aucun compte rendu dans la Revue de Linguistique Romane; il était seulement prévu, mais ce ne s'est jamais réalisé, une «Bibliographie annuelle analytique et critique ... des travaux relatifs à la linguistique romane..., avec appréciations succinctes et avec indication bibliographique des comptes rendus déjà parus ailleurs», et ne s'est pas réalisée non plus la rédaction dans la Revue de «chroniques courantes, signalant et appréciant les ouvrages les plus récemment parus» $(R L i R, 1,1)$. C'est seulement du temps de Gardette, donc à la renaissance de la Société, dans les années 1950, qu'est apparue à la fin de chaque numéro une très courte «chronique bibliographique» se bornant à signaler les livres reçus, avec quelques lignes d'appréciation d'ensemble, ordinairement flatteuses; il s'y mêlait de temps en temps un véritable compte rendu. C'est Straka qui m'a incité à étoffer cette chronique, à partir de 1979. Il n'est pas faux de dire, je crois, qu'elle a contribué au renom de notre revue. Certes le fait n'est pas reconnu par les instances évaluatrices -et l'on comprend bien pourquoi, c'est qu'il y va de leur propre légitimité-, mais un compte rendu demande autant de travail qu'un article, et c'est un travail, non seulement utile au progrès des connaissances et à la diffusion de la science, mais aussi formateur pour qui l'effectue, que celui qui consiste à se frotter aux productions intellectuelles d'un autre savant, d'entrer dans sa logique, de la faire comprendre avec un regard critique, bref de faire œuvre d'évaluateur. A l'heure où des technocrates nous disent comment évaluer un travail scientifique, doubledécimètre, barème de points et machine à calculer à la main, il est bon de rappeler qu'il existe depuis longtemps une autre façon d'évaluer un travail scientifique; elle consiste à le lire attentivement, la plume à la main (comme on disait), et à dire publiquement ce qu'on en pense. Ainsi pratiqué, le compte rendu est une façon de peser sur une discipline et nos prédécesseurs l'avaient bien compris. Dans les débuts de nos études, les comptes rendus tenaient un grand rôle. Par exemple la Zeitschrift für romanische Philologie, la Romania, la Revue des langues romanes rivalisaient d'ardeur dans leurs comptes rendus, souvent polémiques. Les noms de G. Paris, d'A. Mussafia, de P. Meyer, d'A. Tobler, de M. Grammont, d'A. Thomas, de G. Bertoni sont ainsi attachés à des comptes rendus, qu'on cite toujours, même si les ouvrages recensés par eux sont maintenant périmés.

Je n'ai pas présenté ce bref historique pour regretter le bon vieux temps. Une revue doit s'adapter aux mutations de son époque. Grâce à sa jeune équipe dirigeante, la Revue 
de Linguistique Romane le fait constamment. Mais le fait d'avoir une longue histoire et d'être toujours vivante, comme le prouve un Congrès vivant réunissant plus d'un millier de chercheurs, lui donne le devoir d'œuvrer pour l'illustration et le développement des études romanes, mais aussi le droit d'être écoutée avec attention par des décideurs autoproclamés. $\mathrm{Au}$ total, avant d'être une revue scientifique, ce qu'elle est incontestablement, la Revue de Linguistique Romane est au service d'une idée, la latinità, selon la belle formule «Razze latine non esistono: ... esiste la latinità», qui est inscrite au fronton de notre couverture. ${ }^{3}$

\section{Histoire d'une revue de romanistique comparative: la Zeitschrift für romanische Philologie (par Günter Holtus)}

Les revues spécialisées représentent un forum qui informe de la façon la plus actuelle et la plus continue possible sur le développement scientifique d'une discipline. Dans ce sens, elles représentent un indicateur sensible de l'état des recherches et elles contribuent notablement à leur développement (cf. Schweickard 2003 et Schrott 2003).

La «Zeitschrift für romanische Philologie» (Revue de philologie romane), fondée par Gustav Gröber en 1877 -un an après la mort de Friedrich Diez-, était considérée comme une revue spécialisée, de caractère purement scientifique au sein d'un nouveau contexte dans lequel la romanistique s'était déjà bien affirmée. Le premier volume contient dans le Prospect un modèle in nuce de la philologie romane, que G. Gröber retravaillerait en détail plus tard dans le Grundriss der romanischen Philologie.

Cette formule, souvent attribuée à G. I. Ascoli, semble empruntée à P. Savj-Lopez (1920: 94), dont le texte complet dit: «Razze latine non esistono; esiste invece qualche altra cosa, infinitamente più delicata e più profonda, più sottile e più tenace -qualcosa che non è razza ma è spirito, che non è materia fisiologica ma luce d'idea: esiste la latinità». Dans le même ouvrage, l'auteur dit aussi [31]: «Noi vedremo, nel capitolo seguente, in che cosa consista l'essenza della romanità: mostreremo che se esiste ancor oggi il latino come una viva realtà spirituale congiungente molti popoli, eredi dell'antica Románia, questa parentela di spiriti e di lingue non va tuttavia confusa, come generalmente si crede, con la parentela di razza. Razze latine non esistono, fuori del piccolo territorio nell'Italia centrale, ove abitarono un giorno i Latini. Esistono invece razze molteplici e diverse, che il latino ha spiritualmente unificato, senza can- cellare l'originaria dissomiglianza del sangue. La Romania non fu un'armonia etnica: fu un fatto storico, un'opera di conquista, venutasi a trasformare a poco a poco in un indistruttibile fatto spirituale, che séguita a vivere ancora un millennio e mezzo dopo che quella conquista andò dispersa, e lungamente vivrà, se ancora possiede così prodigiosi germi di energia quali noi oggi vediamo». Dans ce passage l'auteur s'inspire clairement de l'article liminaire de G. Paris, paru dans le tome 1 de la Romania, dont il cite [28-30] un long passage [les pp. 28-30 de l'article]. Mieux même la formule italienne décalque trois phrases du même article [p. 20]: «La Romania, ou l'union des nations romanes, n'a pas pour base une communauté de race. Quand on parle des races latines on emploie une expression qui manque absolument de justesse: il n'y a pas de races latines». P. Savj-Lopez (1876-1919), qui commença sa carrière à Strasbourg (1899-1902) grâce à G. Gröber, fut ensuite professeur à Catane (1903-15), puis à Pavie. Il était venu s'établir à Paris, en 1917, pour y fonder, en pleine guerre, l'Istituto Italiano, destiné à renforcer les liens culturels entre les deux pays. Romaniste, au plein sens du terme, mais aussi traducteur de Schopenhauer, il avait nettement pris position contre l'Allemagne au cours de la guerre. Et s'il fallait un lien supplémentaire entre Le origini neolatine, ouvrage dont découle Le origini de Tagliavani, qui lui doit beaucoup, et la $R L i R$, on le trouverait à la page $10 \mathrm{du}$ t. 1 de la $R L i R$, où W. Meyer-Lübke mentionne avec des compliments le manuel de Paolo Savj-Lopez. 
À l'occasion du centenaire de la $Z r P$, Kurt Baldinger, successeur de Walther von Wartburg et directeur de la revue durant trois décennies, affirme qu'une division de la discipline s'est produite en fonction des différentes langues (avec les revues spécialisées correspondantes), mais qu'il est d'avis qu'une revue philologique panoramique, qui traite des problèmes de la philologie romane générale et de ses subdisciplines sera toujours nécessaire (cf. Baldinger 1977).

Le successeur de Baldinger fut Max Pfister qui, dans deux contributions distantes de vingt années, met en valeur les mérites des deux éditeurs précédents, Walther von Wartburg et Kurt Baldinger (cf. Pfister 1988: 207). M. Pfister présente aussi une sélection d'articles fondamentaux qui met en relief le rôle significatif de la $\mathrm{ZrP}$ dans la discussion scientifique; il ajoute à cela une synthèse de la rubrique introduite par $\mathrm{K}$. Baldinger et intitulée «Discussion de problèmes actuel».

À partir de l'an 2000, les articles consacrés à la linguistique comparative romane, à la philologie gallo-romane (français et occitan), à la philologie ibéro-romane (espagnol, portugais, catalan) et à la philologie italo-romane (italien, sarde) prédominent; les articles de critique littéraire et d'édition de textes sont moins nombreux. Quelques articles sur le rhéto-roman (romanche, ladin, frioulan) et sur le roumain y figurent aussi, bien qu'en nombre réduit. La rubrique «Discussion de problèmes actuels» s'élargit progressivement (12 articles depuis 2000) et on publie de plus en plus d'articles critiques sur des œuvres d'importance fondamentale. Grâce aux monographies publiées en annexes (plus de 50 volumes depuis 2000) et à la «Bibliographie romane», la $\operatorname{ZrP}$ a consolidé son rôle essentiel pour la philologie romane.

Le domaine thématique de la $\mathrm{ZrP}$ comprend la linguistique romane ainsi que, dans une certaine mesure, la linguistique générale, et, au sein de la sphère historico-littéraire, la littérature romane jusqu'à la Renaissance. Chaque année, quatre fascicules qui forment un total de 780 pages sont publiés.

Les Beihefte de la $\operatorname{ZrP}$ appartiennent aux publications renommées de la philologie romane (360 volumes jusqu'à présent: des monographies sélectionnées, des éditions, des habilitations). Chaque année paraissent environ huit volumes. Tant la direction que la tâche de rédaction de ces projets ont lieu à Göttingen.

La Romanische Bibliographie est publiée chaque année en deux ou trois volumes et représente un supplément à la $Z r P$. Elle est consacrée aux différents domaines de la linguistique et de la critique littéraire (à l'exception de la critique littéraire française). En outre, chaque année paraît un Cédérom avec les données accumulées depuis 1997. La finalité de la $R B$ est de continuer à être fidèle à la possibilité d'une philologie romane générale et comparative. Elle se considère comme une œuvre de référence de la philologie romane face aux bibliographies (linguistiques) générales. Pour cela, un réseau international de spécialistes externes qui résident en Allemagne et à l'étranger a été créé: Berlin (FU, Institut ibéro-américain), Göttingen, Hambourg, Iéna, Aberystwyth (Grande-Bretagne), Bolzano / Bressanone (Italie) et Salamanque (Espagne).

La dernière partie de ma contribution à la Table ronde sur le thème «Les revues romanistes» se réfère à la problématique proposée par l'organisateur de notre réunion, à savoir les problèmes de l'évaluation. J'ai déjà mentionné que, dans le cadre du Supplément à la $Z r P$ (Romanische Bibliographie), un comité d'experts international a été mis en place qui, le cas échéant, peut aider l'éditeur de la $Z r P$ à décider de l'acceptation, du refus ou de la révision d'un texte (Peer Review). Il en va de même pour l'évaluation des ouvrages proposés pour la publication dans la série des Beihefte zur $\mathrm{ZrP}$. 
Depuis peu, un phénomène se développe et semble, comme le souligne Martin Gleßgen, problématique: toute revue est soumise à des évaluations et à des classifications externes qui déterminent en même temps la 〈valeur〉 des chercheurs qui publient dans celle-ci. Dans une discussion préalable, menée entre autres avec mes collègues David Trotter et Hans Goebl, j'ai souligné mon hésitation, n'ayant pas encore pris de décision claire face à trois options possibles dans les relations avec les agences d'évaluation: refuser totalement l'évaluation, adopter un point de vue critique ou proposer une coopération sous conditions. Il est nécessaire, selon moi, d'opter pour une procédure commune, non seulement de la part des directeurs de revues, mais aussi des maisons d'éditions, des associations et des organisations scientifiques. Tout cela exige beaucoup de temps et d'énergie, de telle manière que je me suis contenté d'adopter un point de vue critique jusqu'à présent. J'adopterais un autre point de vue (en particulier pour la relève scientifique) si la publication dans des revues classées «C» devenait dommageable pour les auteurs.

Il va de soi que pour aller plus loin, il faut que l'ensemble des critères appliqués par les agences d'évaluation soient divulgués et que l'identité des membres de leurs jurys soit dévoilée. Je suis relativement méfiant à l'égard de ces agences: l'Agence d'évaluation de la recherche et de l'enseignement supérieur (AERES) est une institution française qui me semble peu obligeante; la légitimité de l'ERIH (European Reference Index for the Humanities) me paraît également douteuse. En définitive, nous ne pouvons ici que procéder à un niveau international.

Permettez-moi, en guise de conclusion, d'attirer votre attention sur les nouvelles directives de la Deutsche Forschungsgemeinschaft $(D F G)$ concernant les subventions pour la recherche scientifique. Matthias Kleiner, président de la $D F G$, a expliqué dans un commentaire qu'à partir du $1^{\text {er }}$ juillet de l'année 2010 , de nouvelles règles concernant les publications seront appliquées dans les demandes de financement, les ébauches de demandes et les rapports de recherches pour la $D F G$. Dans leurs demandes et leurs rapports, les scientifiques devront limiter leurs références à un nombre restreint de publications importantes et pertinentes (cf. Kleiner 2010).

La DFG veut démontrer avec cette nouvelle règlementation que la qualité compte plus que la quantité et que tout repose sur la pertinence scientifique du contenu. Les nouvelles directives concernent deux points-clefs des demandes et des rapports: dans leur curriculum vitae scientifique, les chercheurs ne pourront désormais citer que cinq publications tout au plus -les cinq publications qu'ils jugent eux-mêmes les plus importantes et représentatives de leur travail scientifique-.

Dans la description à proprement parler du projet requis ou déjà traité, il ne sera désormais permis de citer que deux publications par année de la période subventionnée. Si un chercheur fait une demande d'aide individuelle pour trois ans, il aura le droit de citer seulement six publications. Dans le cas de plusieurs demandeurs, trois publications par an tout au plus pourront être indiquées.

Cependant, la $D F G$ ne veut pas seulement limiter le nombre de publications citées et par ce biais réduire le poids des listes de publications. Son but est que la partie principale de la demande ou du rapport regagne en importance, à savoir la description des objectifs que se fixe le demandeur, la description des travaux déjà effectués en amont ou de projets ultérieurs.

Pourquoi donc ces règles aussi restrictives? Depuis plusieurs années, la quantité a pris le dessus sur la qualité en matière de publications scientifiques et de leur évaluation. Cela se remarque dans plusieurs secteurs: dans la distribution de moyens désormais axée sur le rendement, tout comme pour les nominations et les évaluations de demandes de subventions, les indicateurs numériques jouent partout un rôle de plus en plus important. 
On ne se demande souvent plus «Qui a fait des recherches sur quoi?», mais «Où et combien de contributions un auteur a-t-il publiées?». Cela met les chercheurs fortement sous la pression de devoir publier le plus possible. Certes, cette pression existait déjà auparavant. Mais elle n'a cessé d'augmenter et elle s'est à présent établie dans toutes les matières et à tous les niveaux du travail scientifique.

Tout cela nuit à la science en général. Ici il ne s'agit pas d'évolutions propres à l'Allemagne ou à la $D F G$, des mouvements de rejet de cette évolution existent aussi ailleurs.

Je résume: les nouvelles règles, obligatoires pour toutes les demandes, mettent l'accent sur le fait que le nombre de publications citables est à partir de maintenant limité; en même temps, la description à proprement parler du projet scientifique doit gagner en importance c'est-à-dire avoir une plus grande signification. C'est de cette manière que la $D F G$ veut redonner plus de valeur aux contenus des travaux scientifiques et réduire la portée des facteurs purement quantitatifs.

Je suis d'avis que ces directives représentent un progrès pour la discussion scientifique et que nous devrions en tenir compte, dans la mesure du possible, au cours de notre débat sur les problèmes de l'évaluation des revues romanistes.

\section{Estudis Romànics (par Joan Veny)}

Els organitzadors de la taula redona ens demanen als editors de revistes romàniques una reflexió sobre les formes d'avaluació fonamentades i contrastades amb la pròpia experiència. Es tracta d'un tema no gens trivial, entre altres raons perquè pot tenir conseqüències quan es tracta d'avaluar el currículum d'un aspirant a una plaça universitària o a un centre de recerca o bé d'emetre un judici sobre l'obra investigadora del sol-licitant d'un tram d'investigació.

En qualitat de director, juntament amb el prof. Antoni M. Badia Margarit, de la revista Estudis Romànics, publicada per l'Institut d'Estudis Catalans, em permetré de traçar una breu història d'aquesta revista i d'assenyalar-ne algunes característiques, en les seves dues fases de publicació (ER-1: 1947-1987; ER-2: 2000-2010), característiques relacionades sobretot amb els criteris d'avaluació que les agències posen en pràctica.

Una revista no neix en el buit. Hi ha sempre un caliu que abona la seva aparició. En el cas del domini català, la inquietud primerenca per l'estudi de la llengua sorgeix vinculat de bona hora al zel dinàmic i innovador dels països centreeuropeus. Després que la romanística hagués irromput amb prestigioses revistes com Revue des langues romanes (1870), Romania (1872), Romanische Forschungen (1883), Antoni M. Alcover fundà el Bolletí del Diccionari de la Llengua catalana (1901-1926), per tal de tenir contacte amb la xarxa de col-laboradors del Diccionari de la llengua Catalana (futur Diccionari català-valencià-balear), que ell mateix promogué i on van aparèixer articles d'il-lustres romanistes (Meyer-Lübke...). Constitueix la primera revista de filologia de tot l'Estat espanyol; interrompuda el 1926, un deixeble d'Alcover, Francesc de B. Moll, la va continuar el 1933, però va restar estroncada amb el començament de la Guerra Civil (1936).

Cal tenir en compte que el Bolletí és anterior a revistes com Studi romanzi (1903), la Revue de dialectologie romane (1909) o la Revista de Filología Española (1914). El 1913 l'Institut d'Estudis Catalans llançà el Butlleti de Dialectologia Catalana (1913-1937) i a l'entremig 
aparegué l'Anuari de Lingüística i Literatura (1928-1934), publicat per la Biblioteca Balmesiana: notem en el títol l'adjectiu romànica, que ja indica una vocació no limitada a la llengua catalana, on apareix una àmplia secció de bibliografia, dedicada sovint a obres de fora del domini lingüístic i un llarg article de F. de B. Moll, important per a la romanística, com és el Suplement català al Romanisches Etymologisches Wörterbuch.

Ramon Aramon, que redactava la secció bibliogràfica, va rebre justament de la Biblioteca Balmesiana una beca per a ampliar estudis a Leipzig i Berlin, on va gaudir de l'ensenyament d'eminents romanistes com Gamillscheg i Wartburg. Va ser Aramon, peça clau en la restauració de l'Institut d'Estudis Catalans (1942), el fundador d'Estudis Romànics (19471991). La revista va aparèixer en plena època franquista, en la clandestinitat, quan l'Institut no disposava de locals ni de subvencions, publicada amb les aportacions de generosos mecenes. Portada amb un estil personal, cal reconèixer, com ha dit Badia, que, tot i que era la revista d'una llengua perseguida, estava a l'altura de les revistes d'una llengua de vida normal.

Aquesta primera fase de la revista (ER-1) es va interrompre el 1987 per esser continuada sota la direcció de Badia (2000-2002, vol. XXII-XXIV) i, més tard també de Veny (20032010, vol. XXV-XXXII) (ER-2). Comentarem ara algunes característiques de la revista en les seves dues fases i en relació amb alguns dels criteris d'avaluació.

La qualitat de la revista resta assegurada per la categoria científica dels autors, primeres espases en la investigació romànica: Aebischer, Rohlfs, Delbouille, Iordan, Ruggieri, H. Meier, Wartburg, Coromines, etc. en ER-1; Pottier, Chambon, Herman, Muljačić, Alinei, Tavani, Gulsoy, Goebl, C. Pensado, etc. en ER-2.

Tanmateix, entre les dues èpoques de la revista es noten evidentment diferències propiciades per l'evolució dels estudis de lingüística i crítica literària i per la pausa de tretze anys que separa una i altra etapa (1987-2000); així, a una orientació primordialment filològica d'ER-1 succeeix una ampliació de mètodes (fonètica experimental, dialectologia perceptiva, dialectometria, pragmàtica, etc.), sense que hi falti algun article filològic, verament modèlic, com el de Marcos Álvarez sobre la lexia »a [de] toda broza" (ER, XXX, 2008, 55-83).

Quant a les llengües objecte d'estudi, al català, castellà, italià, francès i estudi comparatiu (ER-1), s'afegeix el gallegoportuguès i tenen més paper l'italià, l'occità, el francès i l'aspecte comparatiu (ER-2). Pel que fa a l'aspecte literari, ER-1 es va centrar sobretot en la literatura medieval i la Renaixença, mentre que ER-2 ha ampliat l'àmbit d'estudi a la literatura moderna i contemporània. Com a llengües instrumentals, l'alemany tenia una participació important al costat del català, el castellà, l'italià i el francès en ER-1, però en ER-2 l'elenc s'amplia a l'occità i el gallegoportuguès, mentre que l'alemany només és testimonial i l'anglès absent. A aquest propòsit, vull remarcar la voluntat del Comitè de Redacció d'ER-2 de fomentar i dinamitzar l'ús de les llengües romàniques com a eina de comunicació.

Pel que fa als criteris d'avaluació, és evident que feien falta, tot i ser patent que el model de Ciències s'ha arrossegat a Humanitats, sense tenir en compte les característiques pròpies d'aquest darrer àmbit. Ningú dubta de la necessitat que en una revista constin el nom i filiació institucional dels autors, l'adreça postal i electrònica de la revista, els resums en anglès, etc.; fins i tot és útil, peró no indispensable, que figuri al peu de totes les pàgines de cada article el membret del número de la revista i la respectiva extensió. També és important la periodicitat: en aquest sentit, ER-1 no va respectar sempre l'aparició anual, amb pauses a voltes de tres o quatre anys ( 20 vols. en 47 anys), cosa que amb perspectiva històrica ens guardarem prou de 
criticar ateses les circumstàncies adverses per les quals va passar; en canvi, ER-2 ha estat fins ara escrupolosament complidora en aquest respecte (10 volums en 10 anys).

L'originalitat i qualitat dels articles, en aplicació dels criteris avaluadors, rau en el control per part dels avaluadors externs; em sembla bé, però la qualitat d'una revista no depèn solament d'aquest aspecte; al meu parer, caldria una avaluació global, de conjunt, on, a més dels articles, es tinguin en compte altres seccions de la revista i la manera de cobrir-les: em refereixo a una miscel-lània on es doni compte de les activitats de centres de recerca, el llegat científic d'un romanista, l'estat d'obres en curs, etc.; a una crònica de les activitats desenvolupades els darrers anys en l'àmbit de l'especialitat de la revista; al nombre de recensions i de notes necrològiques; etc.; en suma, caldria donar una nota de conjunt, una puntuació a part per tots aquests aspectes. En aquesta línia, ER-2 ha completat en aquests punts la contribució d'ER-1, limitada a articles i ressenyes. Entre els criteris d'avaluació, no es fa una referència a l'extensió dels articles; potser no cal, però un cert control sembla que s'imposa per tal d'evitar productes d'excessiva llargària (per ex., el de I. Neu-Altenheimer, de 348 pàgs. a ER-1).

Finalment, voldria fer una observació respecte de les revistes considerades de tercera categoria: més d'un estudiós ha estat avaluat negativament a conseqüència d'haver publicat en una d'aquestes revistes simplement per no tenir la categoria 〈A〉 quan la qualitat del seu article era alta. Aquestes revistes són necessàries perquè hi fan les primeres armes els joves investigadors i, sens dubte, hi apareixen articles valuosos; per exemple, dels 300 articles que he publicat, considero que un dels millors va veure la llum en una d'aquestes revistes. Aleshores crec que s'imposa una recomanació: abans de rebutjar un candidat per semblant circumstància, no seria millor analitzar -o fer analitzar- el seu article per a calibrar la seva qualitat? Imagino la resposta, però algú ho havia de dir.

\section{La Revista de Filología Española (par Pilar García Mouton)}

Represento en esta mesa redonda a la Revista de Filología Española (RFE), fundada en 1914 por Ramón Menéndez Pidal como órgano de difusión de los trabajos que la Sección de Filología desarrollaba en el Centro de Estudios Históricos, creado por la Junta para la Ampliación de Estudios cuatro años antes. En los primeros años de la revista sus fascículos trimestrales permitían seguir los avances en los temas en los que la Sección de Filología, con su director Ramón Menéndez Pidal al frente, volcaba sus intereses filológicos para investigar en lengua, literatura e historia los orígenes y el desarrollo de la cultura peninsular identitaria.

Aquella Revista de Filología Española pronto consiguió reconocimiento por su oportunidad, su rigor y la implicación personal de quienes la crearon y la mantuvieron hasta que la guerra civil española y sus consecuencias los dispersaron. Entre 1937 y 1941 la revista se publicó sin nombre de responsable; finalmente Vicente García de Diego aparece como director a partir de 1943, pero hay que esperar hasta 1948 para ver cómo la RFE reemprende su marcha con Dámaso Alonso, que fue el director hasta 1980 cuando la dejó, por razones de edad, en manos de Manuel Alvar -representante de la nueva generación de filólogos de la postguerra- que era entonces el subdirector. Alvar escribió en 1988 en las Romanische Forschungen que, entre los propósitos de la RFE, estaban el de «recuperar a los hombres que, por causas ajenas a la ciencia, 
quedaron separados» de la revista y el de «dar continuidad a la obra de Menéndez Pidal y sus discípulos y heredar, sin ruptura, todo lo que prestigió a la ciencia filológica de España».

He querido hacer un pequeño recorrido por la historia, conocida por muchos, de la Revista de Filología Española porque probablemente no lo sea tanto para los más jóvenes. Recientemente, en el año 2007, el CSIC actual celebró el Centenario de la Junta para la Ampliación de Estudios, la JAE, reconociéndose heredero científico suyo (Puig-Samper 2007). En el ámbito de las Humanidades en España, la Revista de Filología Española, que pronto será centenaria, constituye un caso excepcional de continuidad.

Actualmente el Consejo de la RFE trata de mantener un equilibrio entre el respeto a la tradición heredada y la incorporación de los cambios que los medios actuales permiten, acogiendo trabajos relacionados con todas las filologías englobadas bajo su nombre. El problema de la aparición de revistas nuevas que responden a la compartimentación de nuestra disciplina en áreas parciales, no le ha hecho perder la visión cohesionadora y amplia que ofrece nuestra forma de concebir la Filología.

Esta mesa redonda está enfocada hacia los problemas de evaluación que las revistas científicas han experimentado en los últimos años porque, como bien han expuesto los coordinadores de la mesa, la evaluación -siempre necesaria en las tareas científicas- se presenta en las circunstancias actuales de un modo que puede llegar a afectar el trabajo editorial de las revistas.

Llama la atención cómo, desde fuera, la presión de determinadas instituciones y de circuitos editoriales-académicos globalizados ha impuesto, aplicando políticas externas, un tipo de evaluación para las revistas que afecta a su consideración, a su difusión y, a la larga, a las buenas prácticas exigibles. Sin ir más lejos, el breve recorrido que hemos hecho por la historia de la RFE permite ejemplificar un cambio sustantivo de las mentalidades en la historia de la ciencia. En los primeros años de la RFE, su director y sus redactores -Américo Castro, Miguel Asín, Navarro Tomás, Antonio G. Solalinde, Amado Alonso, Montesinos, Dámaso Alonso, entre otros- hacían en la práctica la revista con sus trabajos, y junto a ellos publicaban destacados colegas extranjeros, como Schuchardt, Meyer-Lübke, Jud, Saröihandy, Wagner, Spitzer o Krüger. Pues bien, los criterios internacionales de evaluación que hoy nos presionan no considerarían excelente una revista con un tanto por ciento tan elevado de páginas escritas por su equipo de redacción; la considerarían una revista endogámica.

Recuerdo que fue a lo largo de los años 90 cuando en el CSIC se empezó a hacer hincapié en la necesidad de adecuar las revistas a unas normas nuevas. Lo cierto es que, por sus propias características, la RFE ya cumplía muchas de ellas, como la internacionalización del Consejo Asesor y la presencia en el Consejo de Redacción de una proporción elevada de colegas de otras instituciones, pero otras costó introducirlas, como las que suponían la presencia sistemática del inglés como lengua científica obligatoria. También recuerdo que, en todo lo que significó adecuar el aspecto externo y algunos aspectos técnicos de la revista, el papel de Adelaida Román, especialista en Documentación Científica del CSIC, resultó fundamental y ahorró muchos sinsabores a las revistas del Consejo.

Gracias a ella fuimos conscientes - quizá antes que otros- de que, si queríamos que la Revista de Filología Española apareciera en los índices más representativos de nuestras áreas, debíamos cumplir una serie de condiciones, casi todas -hay que decirloajenas a su contenido científico. Es verdad que las revistas deben salir con regularidad y tener unas marcas determinadas que favorezcan el trabajo de los bibliotecarios y de los 
bibliómetras, pero de ahí a tener que cambiar el diseño tradicional de una revista y su forma de citar para que sus contenidos se puedan escanear sin abrirla siquiera... En la actualidad la RFE aparece en los principales índices para Humanidades, lo que nos ha obligado a hacer ciertos cambios formales en los últimos años, sin grandes problemas.

Otra cosa distinta es que la calificación de una revista como A+, A, B, C, etc., se base en factores ajenos a nuestra cultura científica, como el <índice de impacto», problemáticos en áreas tan abarcadoras. No son éstos buenos tiempos para la Filología que, desde el punto de vista editorial, tiene menor presencia en los paneles internacionales de evaluación que otras especialidades que, además, tienen núcleos cercanos a los grandes editores y distribuidores de revistas científicas, fundamentalmente de otro tipo de revistas científicas distintas a las filológicas. Es obvio que todos defendemos la evaluación de la calidad, pero no la aplicación ciega de unos criterios que muchas veces calcan los que se aplican -es de suponer que razonablemente- fuera de las Ciencias Humanas. Y esta evaluación de las revistas, que afecta al sistema general de evaluación académica, acaba repercutiendo en qué tipo de investigador se dirige a ellas y en la «cotización〉 que el mercado académico otorga a publicar allí, desvirtuando muchas veces los objetivos puramente científicos de una revista. De este modo, la evaluación puede llegar a convertirse en el problema. Y tanto las revistas como quienes publican en ellas corren el riesgo de invertir en ese proceso una parte importante de las fuerzas que debieran emplear en buscar calidad y originalidad.

En cuanto a la evaluación desde dentro, la que la revista ejerce sobre el trabajo de los investigadores que aspiran a publicar en ella, estoy segura de coincidir con otros de mis colegas al apuntar que esos criterios externos en ocasiones también cohíben decisiones que el Consejo de Redacción y el Consejo Asesor podrían tomar en beneficio de la línea de la propia revista. La línea de una revista, lo que define su personalidad, es un bien intangible que muchas veces cuesta mantener en el contexto de la llamada evaluación por pares, que, por otra parte, la RFE cumple escrupulosamente. Evaluar en el marco de una línea editorial debería ser, en gran medida, responsabilidad interna, porque son esas decisiones las que definen a la larga la línea de una revista. Es cierto que los expertos son los que mejor conocen un ámbito reducido y su ayuda resulta inestimable. También es cierto que se trata de evitar que el poder de decisión de quienes hacen una revista se ejerza de un modo excesivo, pero esos excesos, de darse, se vuelven contra la propia revista, de manera que todos los editores somos conscientes de que hay que evitar el sistema clientelar sin renunciar a los rasgos propios de la revista. Por eso, en lo referente a las reseñas, el Consejo de Redacción de la RFE decide qué obras considera que deben reseñarse y busca a las personas más cualificadas para hacerlo, evitando en lo posible problemas de circularidad.

Resulta obvio que en el contexto actual no beneficia presentar las Humanidades y, dentro de ellas, nuestras revistas como ajenas a criterios objetivables, imposibles de evaluar, atípicas en todo, singulares a ultranza. Necesitamos índices específicos que consideren desde un punto de vista científico las características de estas revistas. En ese sentido, deberíamos adecuarlas a los criterios externos en lo que las beneficie y en lo que no las perjudique, para hacer fuerza conjunta, ser firmes en defender las que sean características irrenunciables. De hecho, en España, instancias como la Comisión Nacional Evaluadora de la Actividad Investigadora (CNEAI) matizaron sus criterios para evaluar nuestras áreas gracias a la resistencia razonada que en su día se opuso. Yo misma coordiné a lo largo del año 2006 un grupo de trabajo 
que elaboró los Criterios de calidad en la investigación en Humanidades, colaboración de la Agencia Nacional de Evaluación y Prospectiva (ANEP) y la Fundación Española para la Ciencia y la Tecnología (FECYT) ${ }^{4}$ que dio lugar al documento de categorización de las revistas de Humanidades ${ }^{5}$. Convendría que, en la medida de lo posible, tratásemos de conseguir índices cercanos y de aumentar la escasa presencia de filólogos en los principales paneles de evaluación, que son los lugares donde se toman decisiones.

\section{Medioevo Romanzo (par Alberto Varvaro)}

Quando parliamo di riviste scientifiche e di problemi di valutazione, è opportuno fare una distinzione netta tra due procedure del tutto diverse: da un lato il giudizio che il direttore o il gruppo a ciò delegato ha sempre fatto degli articoli sottoposti alla rivista, dall'altro la valutazione che nella nostra epoca è determinata da un organismo esterno ed ha serie conseguenze sulla vita dei periodici scientifici.

Il primo tipo di valutazione c'è sempre stato. Le riviste che pubblicavano indiscriminatamente ogni tipo di materiale ad esse offerto non hanno mai goduto di alcuna considerazione. A valutare erano in genere il direttore responsabile della rivista e alcuni esperti da lui richiesti di consiglio. Il prestigio della rivista discendeva in buona parte dal prestigio del direttore e dalla sua severità. Si poteva giungere al caso di G. I. Ascoli, fondatore e direttore dell'Archivio glottologico italiano, che spesso interveniva non solo sulla forma ma sulla sostanza dello studio proposto, per esempio sostituendo all'etimologia proposta dall'autore dell'articolo e da lui giudicata insostenibile una soluzione propria e informandone l'autore solo dopo o inserendo una nota a sua firma nella quale proponeva la correzione al testo. La corrispondenza di Ascoli, in parte pubblicata, mostra che gli autori non erano lieti di questo interventismo, ma non osavano contraddire l'autorevolissimo direttore. D'altronde essere accettati sull'Archivio aveva tutt'altro peso che pubblicare su una rivistina qualsiasi.

Quanto più severa è la valutazione della direzione, tanto maggiore è, presumibilmente, il numero degli articoli respinti. Ma di questo mi pare che non si sappia nulla, né per il passato né per il presente. Eppure un dato assoluto o percentuale sarebbe quanto mai significativo sotto diversi profili e potrebbe essere più utile di altri parametri usati nel secondo tipo di valutazione, di cui parlerò poi.

A proposito di articoli respinti, devo dire per l'esperienza di direttore di Medioevo romanzo per alcuni decenni, che capita ogni tanto di vedere apparire più tardi un saggio respinto su riviste altrettanto o più prestigiose. Di ciò io non mi scandalizzo. Ogni nostro giudizio è inevitabilmente soggettivo e non può pretendere ad un valore assoluto. Ciò che io giudico scadente può sembrare buono ad altri. Non proporrei mai un albo degli articoli respinti, una sorta di casellario giudiziario degli studiosi: molto meglio che ogni rivista sia libera di giudicare pubblicabile quello che altre non approvano. Giudicheranno i lettori.

\footnotetext{
Madrid, FECYT, 2007.

5 Categorización de las revistas españolas de Ciencias Sociales y Humanas según Criterios de calidad en la investigación en Humanidades ( $A N E P / F E C Y T$ ), informe de marzo de 2008 del Grupo de investigación de evaluación de publicaciones científicas del IEDCYT (CCHS-CSIC).
} 
Più utile è dire qualcosa sulla scarsa soddisfazione degli autori per le critiche e le modifiche richieste dai direttori e/o dai loro consulenti. Può darsi che qualcuno ne sia lieto; del resto i caldi ringraziamenti che si leggono nelle prefazioni dei libri di area anglosassone a quanti hanno messo riparo agli errori dell'autore dovrebbero dimostrare che la gratitudine esiste. Nei nostri paesi, però, gli autori sono per lo più fermamente convinti che quanto hanno scritto sia perfetto e quindi intoccabile ed i loro sentimenti verso i critici vanno dall'insofferenza in avanti. Chi sa perché, la debolezza umana fa in genere preferire gli elogi di qualche amico senza prestigio a qualsiasi critica, anche autorevole e giustificata. Il nostro amico Gilles Roques è qui oggi tra di noi in virtù del fatto che la consuetudine del duello (all'arma bianca o all'arma da fuoco e comunque all'ultimo sangue) non ha più la diffusione che aveva un secolo fa, quindi per una semplice questione di moda. Se il pensiero bastasse a uccidere saremmo privi del piacere di ascoltare le sue parole.

Nessuno, o ben pochi, degli interessati sembra convincersi del fatto che la valutazione, anche quella dei recensori, ha un aspetto positivo: chi sopravvive illeso ad una recensione di un critico come Gilles Roques è come se avesse ricevuto una medaglia al valore, un certificato di qualità. Per citare un altro caso ben noto, il grande Yakov Malkiel era recensore molto severo, ma una recensione favorevole di Malkiel era una forte raccomandazione positiva nel caso che il recensito cercasse di ottenere un posto presso una università degli Stati Uniti. Il che significa, molto banalmente, che la valutazione è uno strumento di polarizzazione della qualità.

Il punto chiave è come venga fatta questa valutazione. E qui passo dalla valutazione interna ai periodici scientifici a quella esterna, cui siamo sottoposti da qualche tempo. Se la prima ha un correttivo nella possibilità di avere molteplici valutazioni da differenti riviste, la seconda invece è più pericolosa, perché obbedisce ad un criterio unitario e assoluto e non ammette correttivi.

Non è esatto dire che la valutazione comparativa delle riviste ci è stata imposta dall'esterno e da poco tempo. In realtà tutti abbiamo sempre saputo che la Zeitschrift für romanische Philologie aveva nel nostro campo una qualità molto superiore a quella degli annali di una qualsiasi università di provincia. E tutti abbiamo sempre sperato che i nostri lavori apparissero su riviste notoriamente prestigiose. È solo il recente (da mezzo secolo circa) proliferare delle pubblicazioni in conseguenza di un accresciuto benessere e della esigenza di tali pubblicazioni per la carriera dei docenti che ha attenuato questa graduatoria, che era data per scontata: ora qualsiasi sede diventava equivalente. Ricordo una combattutissima commissione per una borsa di studio, nella mia università, nella quale una pubblicazione da parte di una banca a San Benedetto del Tronto fu considerata sullo stesso piano di una apparsa presso Yale University Press.

Il punto è che, mentre un tempo la valutazione comparativa delle nostre riviste discendeva da un tacito consenso tra di noi, non basato su criteri formali e astratti, oggi la valutazione è fatta da organismi esterni e con criteri predeterminati. È dunque indispensabile che tali organismi siano un po' meno esterni e tali criteri un po' meno calati dall'alto, senza per ciò venir meno alla correttezza. Per ottenere questo dobbiamo contare di più nelle sedi nazionali ed internazionali, specialmente in quelle dell'Unione Europea, non per chiedere e ottenere privilegi ma per quel tipo di lobbying che è del tutto morale e lecito, cioè perché sia chiaro quali sono le nostre specificità ed esse siano tenute in conto. È gravissimo che negli schemi europei non esista la casella 〈filologia〉, ma solo quelle 〈linguistica〉 e «letteratura〉. In secondo luogo è ovvio che per noi una valutazione in base a dati puramente quantitativi è inadeguata. In terzo luogo è 
indispensabile che la valutazione non consideri solo fini pratici e, come è stato detto, non privilegi a priori l'insegnamento dell'inglese rispetto ad uno studio, di valore scientifico pari o superiore, di un dialetto dell'Alta Ribagorza. In terzo luogo, deve essere chiaro che la nostra 〈filologia〉 può essere declinata in accezioni diverse non solo rispetto all'articolazione geolinguistica ma anche, e forse soprattutto, a quella metodologica. Nel nostro campo, con ragione e per fortuna, non esiste una dottrina unitaria (mentre invece non mi pare che esistano fisiche atomiche alternative). Basti considerare quanto siano diverse tra di loro tre fra le maggiori riviste del settore, la già citata Zeitschrift für romanische Philologie, Romania e la Revue de linguistique romane.

$\mathrm{Su}$ questi aspetti si deve e si può intervenire. Non con inutili piagnistei e recriminazioni, ma costruendo il più vasto consenso possibile tra di noi e con i settori che ci sono vicini, possibilmente tra tutte le scienze umane, e poi imponendone la considerazione agli organi decisionali esterni, quelli politici. Si tratta di un lavoro lungo, paziente e difficile, ma inevitabile. Io credo che esso possa essere portato al successo.

\section{Les revues de romanistique et le monde anglophone (par David Trotter) ${ }^{6}$}

Les questions de l'anglais et de l'univers scientifique anglophone, questions qui pourraient sembler extérieures à notre débat, $\mathrm{y}$ occupent pourtant une position particulière, et cela pour plusieurs raisons.

Primo, parce que l'évaluation dite «scientifique», et qui dépend d'un anonymat somme toute frauduleux (des deux côtés), est quasi-universelle dans le monde anglophone. On y tient. Le monde anglophone l'a imposée mais sans être toujours conscient des insuffisances du système. Il y a lieu de se demander d'une part si l'évaluation est réellement anonyme, ce que je ne crois pas -Martin Glessgen explique pourquoi-, et surtout d'autre part si elle est efficace, c'est-à dire si elle garantit la qualité des contributions. Là encore, il est permis d'en douter. Il suffit d'entendre ce que disent à ce propos nos amis et collègues, rédacteurs des grandes revues romanistes telles que la Zeitschrift ou la Revue de Linguistique Romane. Si on évaluait le niveau des articles publiés dans les grandes revues, ce qui devrait être en réalité la finalité de toute cette démarche, je ne crois pas que le système qui consiste à faire confiance aux directeurs soit moins efficace que celui qui implique des 〈Peer Reviewers〉. Mais les anglo-saxons, comme dirait De Gaulle, n'acceptent pas que le directeur dirige, et qu'il ait cette responsabilité. Ils sont attachés au système qui se base sur une évaluation supposée objective et anonyme.

Cette attitude doit être replacée dans le contexte de l'évaluation de la recherche universitaire portant sur la production scientifique de chaque institut et de chaque université, dans toutes les disciplines. Celle-ci fait partie depuis maintenant trente ans du paysage scientifique de la Grande-Bretagne. Les procédés de l'AERES, par exemple, n'en sont qu'un pâle reflet. Le processus tel qu'il est pratiqué Outre-Manche, basé sur l'évaluation d'un nombre restreint de publications (point fort de ce système), a d'ailleurs des conséquences financières réelles: un institut jugé défaillant peut perdre des sommes faramineuses et risque même, dans la situation actuelle, de disparaître. Les enjeux

6 Pour laisser la place à la discussion avec la salle, la présente contribution, préparée et prévue pour la Table ronde, n'a pas été lue à Valence. 
sont donc importants. C'est une évaluation sérieuse mais également très coûteuse. Les responsables souhaiteraient en réduire le coût: un système de classement des revues aiderait peut-être, s'il permettait de court-circuiter l'opération qui consiste à lire et à évaluer chaque article publié.

Secundo, il ne faut pas oublier la présence disproportionnée des revues anglophones dans les listes créées par les agences internationales telles l'ERIH ou l'AERES. Cela, en dépit du fait que les anglophones (c'est-à-dire mes compatriotes britanniques) ont été parmi les premiers à dénoncer les listes dites 〈européennes〉. Non pas que nous soyons contre l'Europe (comme pourrait le faire croire la lecture de certains quotidiens de l'île), mais plutôt que la quasi-élimination de tout ce qui n'est pas rédigé en anglo-saxon nous paraisse inconcevable dans un contexte européen. Ont protesté non seulement les romanistes, qui sont directement impliqués, mais aussi les spécialistes des langues celtiques, les historiens, les anthropologues, les archéologues.

L'évaluation est donc, en Grande-Bretagne, une réalite omniprésente, et souvent dangereuse. Omniprésente dans ses revues, qui jouissent d'une importance excessive dans les listes 〈européennes〉; omniprésente aussi dans l'évaluation de la recherche au sein des universités, à tel point qu'on se demande parfois si l'évaluation ne prend pas plus de place que la science. En tout cas, le fait d'être évalué tous les cinq ans a certainement contribué à l'idée que tout 〈projet〉 doit être bouclé assez rapidement.

Mais ici, au lieu de simplement déplorer ces processus et leurs préjugés sous-jacents, il est utile, voire important, de s'interroger sur la genèse de ces listes et sur le rôle de l'évaluation. Le point de départ des deux opérations se trouve dans les sciences dures dont les résultats voient le jour (on le sait) essentiellement en anglais. Ici le quantitatif joue un rôle important: un scientifique travaillant dans la discipline des biosciences peut avoir affaire chaque année à un million d'articles publiés dans des revues sérieuses. Certains professeurs embauchent des assistants qui ont pour tâche principale de lire les résumés et de leur signaler ensuite les articles (ou peut-être seulement les résumés) à connaître ou à lire autant pour se tenir au courant que pour alimenter leur propre recherche. Depuis longtemps, les revues dans les sciences dites 〈exactes〉 ont une classification interne qui attribue à chaque revue un 〈facteur d'impact), lui aussi très... 〈exact〉 et basé sur de multiples éléments (permettant ainsi de fournir des résultats du type «5,71»). C'est un moyen, peut-être scientifique, du moins qui veut l'être, de faciliter le parcours du combattant qui consiste à s'informer sur ce qui se passe et à évaluer les études de plus en plus nombreuses. Ces chiffres sont ensuite disponibles à d'autres fins: le recrutement d'un enseignant-chercheur ou la décision d'attribution d'un soutien financier.

Or, c'est la recherche en sciences exactes qui coûte le plus ou en tout cas qui consomme la plus grande quantité d'argent, d'où son rôle de meneur du jeu. Il est important -pour des raisons bassement financières mais aussi hautement scientifiques- que la recherche soit évaluée avant et après son exécution, c'est-à-dire: avant d'être financée et, après exécution, avant la publication des résultats. Les enjeux sont bien sûr autrement plus importants qu'en romanistique: une fausse étymologie agace, certes, mais ne tue pas, tandis qu'une erreur dans la recherche médicale ou pharmaceutique met souvent des milliers de vies en danger.

Mais -et c'est la preuve ici que tertium datur- quiconque a revu la liste de l'ERIH l'aura remarqué, il y a un préjugé, provenant également à mon avis de la domination exercée par les sciences exactes, qui fait que l'anglais est roi. En sciences exactes, c'est un fait avéré pour 
ne pas dire exact. En romanistique par contre, et pour un romaniste anglophone, la réalité est affligeante: il y a trop de revues relativement négligeables mais rédigées en anglais qui sont classées $\langle A\rangle$ tandis qu'on note dans cette classe supérieure l'absence honteuse et franchement ridicule de pratiquement tout ce qui compte dans notre discipline mais qui est publiée dans une langue autre que l'anglais. Cette liste a un impact réel car beaucoup de chercheurs vont la respecter, parce que leurs universités y croient (malheureusement) et y tiennent (bêtement). Publier dans la liste 〈A〉, c'est l'équivalent d'être sur la liste 〈A〉 si on est actrice. S'ajoute à cela bien entendu la publication en linguistique générale qui a tendance à s'effectuer elle aussi en anglais, et pour laquelle il existe de nombreuses revues scientifiques dont la majorité publie en anglais. Tout cela pose un problème particulièrement délicat pour les romanistes et surtout, pour les romanistes anglophones.

Car siempre la lengua fue compañera del imperio. C'est indiscutablement le cas pour l'anglais de nos jours. Si l'allemand, jadis Fachsprache der Romanistik (et sans la connaissance de laquelle il reste quand même difficile d'envisager de travailler de manière sérieuse en romanistique), disparaît petit à petit des revues, je ne vois nullement pourquoi l'anglais devrait le remplacer. Il y a une quantité d'études incontournables publiées en allemand. L'anglais ne peut pas se vanter d'une histoire aussi glorieuse. Son prétendu statut de langue scientifique internationale n'y est pour rien dans notre discipline. La valeur intrinsèque de l'anglais (en romanistique) est difficile à comprendre. Il ne sert pas à la diffusion globale d'un savoir dans un champ d'études qui se base sur la connaissance des langues romanes. Une publication portant sur l'aragonais ou sur le vénitien, en anglais et rédigée par un non-anglophone, me semble pour le moins discutable. Pour une raison bien simple: il n'est pas possible d'être spécialiste de l'aragonais sans connaître l'espagnol. Un scientifique qui s'intéresse au vénitien ne peut pas ignorer l'italien. Publier en anglais, n'a pas de sens dans ce contexte. Autre phénomène curieux, voire excentrique: une revue comme le Journal of French Language Studies, ouvert à des articles en anglais ou en français, devient de plus en plus francophone. Pourquoi ? Peutêtre parce qu'il est désormais classé 〈A〉 dans la liste de l'AERES, en France. Tout est bien qui finit bien, comme le dirait Shakespeare? Non, car l'AERES continue à classer $\langle\mathrm{B}\rangle$ ou même (C) la plupart des grandes revues de la romanistique, y compris Medioevo Romanzo, Romance Philology, Romania, et continuant tranquillement à se tromper, sans gêne, jusqu'à la fin de l'alphabet avec la Zeitschrift für romanische Philologie. On se retrouve dans la situation de Groucho Marx, qui ne voulait pas être membre d'un club qui accepterait de l'admettre.

En tout cas, il n'est pas logique que la langue d'une revue -surtout, s'il ne s'agit pas de la langue qu'on décrit ou qu'on étudie-détermine a priori le statut accordé à un article, ou pire encore, le statut de la revue elle-même. La romanistique est avant tout plurilingue: les contributions à ce débat expliquent pourquoi. La raison d'être de la romanistique est d'ailleurs de confronter toutes les langues romanes. Il est complètement aberrant que la publication en anglais prime sur la publication en italien, en français, en espagnol. Dans la mesure où les revues anglophones souhaitent être présentes dans la romanistique internationale, il me semble impératif que les langues romanes y soient plus visibles. 


\section{Interventions}

\subsection{Pietro Beltrami}

Va preso molto sul serio un problema che è stato enunciato nelle presentazioni, e cioè che nelle varie griglie disciplinari che vengono usate per classificare i progetti, le domande di finanziamento, le relazioni di valutazione ecc. sta scomparendo, se non è già del tutto scomparsa, la parola «filologia»: non «filologia romanza», ma proprio «filologia», in qualunque sua specificazione; ciò che ha a che fare con la filologia si deve necessariamente iscrivere o sotto «linguistica» o sotto «letteratura». Ciò è scientificamente inaccettabile, ed è dannoso sul piano pratico per chi presenta progetti o deve essere valutato. Su questo punto tutti i filologi che ne hanno la possibilità dovrebbero attivarsi con la massima energia.

Per quanto riguarda i criteri di valutazione, va reso noto che il Consiglio di Amministrazione del Consiglio Nazionale delle Ricerche (CNR), il maggiore ente di ricerca italiano, ha recentemente approvato un documento prodotto da una commissione dell'area umanistica, nella quale si sostiene che l'area umanistica deve essere valutata con criteri diversi da quelli in uso per l'area scientifica, ha negato validità all'impact factor e alla catalogazione ISI e proposto una serie di possibili criteri da sperimentare. ${ }^{7}$ Non si può ancora dire a che cosa ciò porterà, ma questo è un incoraggiamento a proporre e a cercar di fare adottare criteri di valutazione per le scienze umanistiche più appropriati di quelli che ci vengono attualmente imposti.

\subsection{Lino Leonardi}

Ritengo, come molti, che la valutazione delle riviste in base a parametri generali, che prescindono dalla valutazione dei singoli articoli, sia una procedura ad alto rischio, nella misura in cui è finalizzata a fornire uno strumento di valutazione della produzione scientifica demandata a criteri non di merito. In particolare desta preoccupazione l'applicazione di criteri bibliometrici derivanti dalle scienze dure anche al settore umanistico. Questo tentativo, da quando sono apparse le prime liste ERIH, è stato da più parti discusso, quando non rifiutato ${ }^{8}$, e il dibattito che si è acceso in molti settori ${ }^{9}$ ha provocato un rinvio della procedura de parte di ERIH, che però dovrebbe produrre le liste aggiornate entro la fine del 2010.

È auspicabile che anche nell'ambito della romanistica (filologia e linguistica) si intraprendano iniziative al fine di far valere criteri e parametri meno alieni dalla natura della nostra attività di ricerca. Penso che su alcuni punti sia possibile trovare facilmente un consenso molto largo: inserimento della filologia come settore autonomo accanto a linguistica e letteratura, pluralità linguistica nella redazione degli articoli, presenza di recensioni critiche, ecc. Inoltre, rispetto alle scienze esatte la produzione scientifica nei nostri settori è meno esclusivamente vincolata alle pubblicazioni su rivista, e si realizza spesso in volumi (monografie e edizioni critiche), la cui valutazione implica necessariamente un esame

Cfr. www.articolo33.it/documenti/Delibera_128_2010.pdf

8 Cfr. p. es. «Journals under Threat: A Joint Response from History of Science, Technology and Medicine Editors», Medical History 53 (2009), 1-4, www.ncbi.nlm.nih.gov/pmc/articles/ PMC2629173

9 Cfr. «Évaluation de la recherche en SHS» http://evaluation.hypotheses.org 
di merito. Propongo che la SLiR si faccia promotrice di un documento da sottoporre a tutte le riviste e le associazioni nazionali di romanistica, in modo da raccogliere una vasta adesione su una linea comune di intervento.

\subsection{Hans Goebl}

Ma prise de position est nourrie de mes expériences faites au cours de mes activités au sein de la Fondation nationale autrichienne pour la recherche scientifique (FWF - Fonds zur Förderung der wissenschaftlichen Forschung) entre 2000 et 2008. ${ }^{10}$ Mais il ne fait aucun doute que l'évaluation -pour ne pas dire l'évaluationite- est de date plutôt récente et a pris, à l'instar (et avec les apparences) d'une épidémie, un essor à la fois rapide et mondial. En tant que linguistes il est bon de se poser le problème de la différence sémantique et pragmatique entre la bonne vieille critique scientifique et la mode récente de l'évaluation. Bien qu'il y ait entre les deux un certain nombre de parallélismes et d'intersections, leur but sociétal est différent. La critique scientifique classique vise le contrôle et le maintien de standards méthodiques et terminologiques (et autres) de la discipline respective tout en étant exercée par d'éminents représentants de cette discipline dont la responsabilité ne touche que la fortune de cette dernière.

L'évaluation moderne va au-delà des limites des différentes disciplines. Elle 〈politise〉, 〈économise〉 et 〈mondialise〉 la critique scientifique classique tout en la dénaturant profondément. Je m'explique:

\section{(1) Ad 〈politisation〉:}

L'évaluation se fait dorénavant dans un contexte politique. Il s'agit de justifier, pour les besoins de la politique universitaire, locale, régionale, nationale et souvent aussi mondiale, telle méthode de recherche, tel investissement (matériel ou «humain〉) tout en établissant des hiérarchies d' 〈utilités〉 et de 〈saillance〉. Ceci entraîne les scientifiques dans des conflits voire des tourbillons de conscience ou de responsabilité pratiquement insolubles.

\section{(2) Ad «économisation〉:}

Ici, il s'agit moins de l'éternel manque de moyens financiers dont nous avons toujours souffert, mais de l'irruption, dans le monde des sciences, d'us et coutumes courants dans le monde de l'économie. Dorénavant la gestion de nos affaires doit être assurée par les impératifs du monde des fabricants, commerçants et banquiers. N'oublions pas non plus que ceci nous est imposé sous le nom du 〈progrès〉 et de la 〈mondialisation〉.

\section{(3) Ad «mondialisation»:}

Nul ne sait mieux qu'un romaniste que le monde ne sera jamais un et homogène. C'est d'ailleurs une banalité pour un scientifique qui, comme observateur attentif de ce qui se passe autour de lui, tire de l'analyse des différences observées la raison d'être de sa propre profession. L'histoire de l'humanité est riche en tentatives unificatrices, souvent naïves, parmi lesquelles je ne cite que le sprocessus de Bologne> dont

10 Les lignes qui suivent constituent un résumé approximatif d'une intervention spontanée faite à la suite du discours d'ouverture du Secrétaire de la SLiR et des interventions de plusieurs éminents confrères. 
l'indéniable échec ne cesse de nous causer tant de soucis. L'effet niveleur qui émane de la mondialisation est tout particulièrement dangereux pour les sciences humaines qui, à la différence des sciences naturelles, tirent toute leur substance de la bigarrure intrinsèque du tissu culturel et social de cette planète. Il ne sera jamais possible de 〈fusionner〉 -pour l'amour d'une plus grande rentabilité ou efficacité- la tibétologie et la romanistique. Et encore: vouloir gérer suivant (exactement) les mêmes principes éditoriaux une revue d'archéologie et une revue de romanistique n'est pas faisable. Ceci n'empêche que de telles tentatives sont à l'ordre du jour dans de grandes entreprises éditoriales, parfaitement 〈mondialisées〉.

Dans la conjecture moderne, la Société de Linguistique Romane et sa Revue sont les 〈habitants d'une niche〉. Pour leur survie -que nous voulons tou- elles ont besoin de la possibilité de se soustraire, dans la mesure du possible, aux trois facteurs mentionnés cidessus. Mon conseil est donc de ne pas nous lasser de cultiver le meilleur de nos bonnes traditions: le plurilinguisme roman, la pluralité des méthodes et points de vue scientifiques et l'indépendance administrative de l'organisation de notre société.

\section{Synthèse}

La question se pose à présent dans quelle direction nous devrions aller. Indépendamment des implications politiques, idéologiques ou stratégiques, une évaluation externe dans les Sciences de l'Homme s'avère extrêmement épineuse d'un point de vue épistémique. La ponctualité de la parution d'une revue, un travail soigné de relecture et de mise en page, la cohérence thématique des contenus, la capacité d'attirer des travaux novateurs, sont certainement des éléments de qualité. Mais en dehors des éléments extérieurs comme la ponctualité et la mise en page, même ces paramètres basiques sont difficiles à cerner, plus encore à hiérarchiser dans la comparaison de plusieurs revues, et impossibles à quantifier.

Gilles Roques et Pilar García Mouton soulignent le caractère fortement individuel d'une direction de revue réussie et le manque de pertinence de certains critères considérés comme essentiels par l'évaluation 〈externe`: comment soumettre le travail d'un maître à un processus de relecture anonyme? En quoi le nombre d'articles rejetés par la revue est-il un signe de qualité si d'eux-mêmes les auteurs tendent à ne pas lui proposer des travaux moins achevés? Pourquoi une revue qui comporte de nombreux articles révisés par un comité de rédaction de qualité serait-elle mauvaise?

Par ailleurs, G. Roques introduit l'argument, souvent négligé, du potentiel identificateur d'une revue pour la communauté scientifique, argument repris par Joan Veny qui ajoute celui de l'importance de la diversité dans l'univers des revues: la 〈valeur〉 d'une revue peut donc justement résider dans ses ambitions restreintes volontairement ou du fait des circonstances.

Günter Holtus met l'accent sur le manque de transparence voire de sérieux des agences d'évaluation qui s'auto-proclament légitimes et capables de parvenir à des résultats pertinents dans un domaine d'une extrême complexité. La multitude des paramètres à prendre en compte dans une évaluation ressort de la mise au point de Joan Veny et de P. García Mouton. S'ajoute le paramètre du choix de langue, notamment dans la décision pour ou contre l'anglais qui s'avère devant les réflexions de David Trotter être un paramètre évaluatif peu fondé. 
Fondamentalement, G. Holtus et J. Veny plaident comme L. Leonardi avant tout pour une prise en considération de la qualité des contenus des publications et des projets de recherche individuels -éléments le plus souvent absents des entreprises d'évaluation externe. D'après G. Holtus, l'intégralité de la communauté des chercheurs devrait faire front devant ce danger pour la Science, danger qui, d'après J. Veny, ne devient que trop réel pour des individus dont la carrière est mise en question par les choix des évaluations externes.

L'ultime question reste alors celle de savoir comment s'opposer à une évolution indiscutablement négative. Toute initiative concrète est certainement utile, comme le soulignent P. García Mouton, A. Varvaro ou L. Leonardi. En même temps, nous sommes en face de tendances idéologiques fortement irrationnelles, ce qui ressort notamment de l'argumentation de Hans Goebl. Il est en effet difficile d'opposer la raison à son absence. Par ailleurs, répétons-le, la complexité des interrogations en jeu est extrême et les problèmes ne s'arrêtent pas à l'évaluation externe du genre scientifique en question. De la même manière que la «conscience romaniste〉 est fondamentale pour nos études, mêmes monolingues, une conscience épistémique commune de la non-validité des évaluations actuelles saura porter ses fruits.

\section{Bibliografia}

Baldinger, Kurt (1977): Hundert Jahre Zeitschrift für romanische Philologie. In: id. (ed.): Beiträge zum romanischen Mittelalter. Sonderband zum 100jährigen Bestehen [der ZrP]. Tübingen: Niemeyer, VII-XIV.

Kleiner, Matthias (2010): Qualität statt Quantität. In: Forschung, Das Magazin der Deutschen Forschungsgemeinschaft 1, 2 sq.

Pfister, Max (1988): Zeitschrift für romanische Philologie. In: RF 100, 332-339

- (2007): Kurt Baldinger (17. November 1919 - 17. Januar 2007). In: ZrP 123, 820-826.

Puig-Samper, Miguel Ángel (ed.): Tiempos de investigación. JAE-CSIC, cien años de ciencia en España. Madrid: CSIC.

Savj-Lopez, P. (1920): Le origini neolatine. Milano.

Schrott, Angela (2003): Romanistische Sprachgeschichtsforschung: Zeitschriften. In: RSG 1, 421-427 (art. 38).

Schweickard, Wolfgang (2011): Romanistische Fachzeitschriften / Panorama des revues romanes. In: LRL 1/2 1186-1194 (art. 90c). 